Revue d'histoire de l'Amérique française

REVUE D.HISTOIRE DE L'AMÉRIQUE FRANÇAISE

\title{
L'exploitation des forêts publiques au Québec (1874-1905) : transition et nouvel essor
}

\section{Guy Gaudreau}

Volume 42, numéro 1, été 1988

URI : https://id.erudit.org/iderudit/304648ar

DOI : https://doi.org/10.7202/304648ar

Aller au sommaire du numéro

Éditeur(s)

Institut d'histoire de l'Amérique française

ISSN

0035-2357 (imprimé)

1492-1383 (numérique)

Découvrir la revue

Citer cet article

Gaudreau, G. (1988). L'exploitation des forêts publiques au Québec

(1874-1905) : transition et nouvel essor. Revue d'histoire de l'Amérique française, 42(1), 3-26. https://doi.org/10.7202/304648ar
Résumé de l'article

Cet article établit l'évolution des récoltes de matière ligneuse sur les terres publiques québécoises entre 1874 et 1905 . Au cours de cette période, deux phases ont été identifiées : une phase de consolidation, au cours de laquelle le volume des récoltes plafonne, et une phase de forte croissance, grâce à l'essor rapide du bois à pâte. Nouvelle manne du secteur forestier, cette dernière catégorie de bois relance les activités affectées par une crise dans l'industrie du sciage. Cette crise frappe certaines régions dès les années 1870 et 1880 , soit beaucoup plus tôt qu'on ne l'avait d'abord cru. La variété des performances régionales constitue par ailleurs l'un des thèmes majeurs de cette étude. C'est pourquoi nous en avons cherché les causes. 


\title{
L'EXPLOITATION DES FORÊTS PUBLIQUES AU QUÉBEC (1874-1905): TRANSITION ET NOUVEL ESSOR ${ }^{1}$
}

\author{
GUY GAUDREAU \\ Département d' histoire \\ Université Laurentienne
}

\begin{abstract}
RÉSUMÉ
Cet article établit l'évolution des récoltes de matière ligneuse sur les terres publiques québécoises entre 1874 et 1905 . Au cours de cette période, deux phases ont été identifiées: une phase de consolidation, au cours de laquelle le volume des récoltes plafonne, et une phase de forte croissance, grâce à l'essor rapide du bois à pâte. Nouvelle manne du secteur forestier, cette dernière catégorie de bois relance les activités affectées par une crise dans l'industrie du sciage. Cette crise frappe certaines régions dès les années 1870 et 1880 , soit beaucoup plus tôt qu'on ne l'avait d'abord cru. La variété des performances régionales constitue par ailleurs l'un des thèmes majeurs de cette étude. C'est pourquoi nous en avons cherché les causes.
\end{abstract}

\section{ABSTRACT}

This article analyses the evolution of the forest industry on Quebec public lands between 1874 and 1905. Two phases have been identified: the period between 1874 and 1890 when the industry encountered difficulties in increasing its output; and the following fifteen years which were characterized by a strong growth in the level of production as a result of the new demand for pulpwood. The crisis in the sawmill industry, which began in some regions as early as the 1870 's, demonstrates clearly the importance of this new forest product. Finally, a major theme of the study is the regional disparity in the rate of development of the forest industry in Quebec and an analysis of the causes of this disparity.

Peu de travaux ont été consacrés à la coupe de matière ligneuse au Québec et au Canada. Jusqu'à tout récemment ${ }^{2}$, seuls les ouvrages de

1 Cet article s'inspire d'une thèse de doctorat soutenue à l'Université du Québec à Montréal en 1986 (L'exploitation des forêts publiques au Québec (1842-1905): cadre juridique, mode d'appropriation et évolution des récoltes). Nous tenons à remercier les directeurs de cette thèse pour leurs remarques et leurs encouragements: Alfred Dubuc, Paul-André Linteau et Normand Séguin.

2 Depuis les publications du groupe de recherche sur la Mauricie, cette lacune a été partiellement comblée. Voir René Hardy, Normand Séguin et al., L'exploitation forestière en Mauricie. Dossier statistique: 1850-1930 (Trois-Rivières, Groupe de recherche sur la Mauricie, 4 (1980); Normand Séguin et René Hardy, Forêt et société en Mauricie (Montréal, Boréal Express, 1984). 
A. R. M. Lower ${ }^{3}$, historien de la forêt canadienne, jetaient un éclairage sur le sujet. Toutefois, celui-ci avait mis l'accent sur les exportations de bois et n'avait donc pas touché directement à la question de la production forestière. Tributaire d'une grille d'analyse du développement économique qui privilégie les marchés extérieurs, le staple, le choix de Lower paraît maintenant insuffisant. C'est une tout autre interprétation de l'évolution du secteur forestier qui se dégage d'une étude qui met l'accent sur la production de bois en tant que critère de périodisation et d'analyse. Ce changement de perspective permet notamment de mettre en lumière le rôle du marché intérieur dans le développement de l'économie canadienne.

D'une certaine façon, nous voulons poursuivre le travail de pionnier effectué par François-Albert Angers il y a plus de 40 ans $^{4}$. Reconstituer l'évolution de la production de matière ligneuse représente une étape essentielle dans la connaissance du secteur forestier. Étape amorcée par Angers avec beaucoup d'ingéniosité, mais qu'il faut approfondir. En identifiant les principales productions, leur importance et leur évolution, et plus globalement le rythme de croissance ou de décroissance du secteur forestier, on jette ainsi les bases des études futures. Ce choix cependant fixe une contrainte: la seule utilisation des données relatives aux forêts publiques. L'ensemble du secteur forestier comprend une exploitation des terres privées que nous n'avons pas pu retenir faute de données annuelles. Cette contrainte provoque assurément des distorsions qu'il ne faut pas perdre de vue: tenir compte de l'ensemble de la production - s'il était possible - entraînerait des modifications dans l'importance respective des différentes catégories de bois.

Les trente années d'exploitation des forêts publiques examinées ici sont marquées par l'émergence du bois à pâte, à l'aube du $20^{\mathrm{e}}$ siècle. Mais, avant son apparition, l'industrie traverse des années d'hésitation qui s'amorcent avec la crise de 1874-1879 et se prolongent durant toute la décennie suivante; par contre, de 1890 à 1905 , la production connaît un renouvellement. Si deux phases bien distinctes tissent l'évolution du secteur forestier québécois entre 1874 et 1905 , on y observe néanmoins une continuité fondamentale: dans les deux cas, l'industrie du sciage présente des signes indéniables de difficultés. Ce malaise permet de mieux saisir le sens de l'arrivée du bois à pâte, la nouvelle manne du secteur forestier québécois.

3 Voir «The Trade in Square Timber», W. T. Easterbrook, M. J. Watkins, dir., Approaches to Canadian Economic History. A Selection of Essays (Toronto, McClelland and Stewart, 1967), 28-48; The North American Assault on the Canadian Forest. A History of the Lumber Trade Between Canada and the United States (Toronto, Ryerson Press, 1938); Great Britain's Woodyard: British America and the Timber Trade, 1763-1867 (Montreal, McGill-Queen's University Press, 1973).

4 Voir «Documentation statistique», in Esdras Minville, dir., La forêt (Montréal, Fides, 1944), 337-404. 
L'analyse qui suit épouse ce découpage en deux périodes et sera suivie, dans une troisième partie de l'étude, des activités forestières à l'échelle régionale. Il faut observer toutefois que cette analyse est conditionnée en bonne partie par un traitement ambitieux des données brutes utilisées. Afin de ne pas alourdir le texte, nous avons reporté en annexe certaines opérations, tandis que d'autres ont déjà fait l'objet d'un volume qu'on voudra bien consulter ${ }^{5}$. Rappelons simplement que les données originales ont subi de profondes transformations afin d'être ramenées à une unité de mesure homogène et continue, et afin de corriger les données défectueuses.

\section{1 - UNE PÉRIODE DE TRANSITION: 1874-1890}

Les années 1874 à 1890 s'insèrent dans une phase historique identifiée par plusieurs comme celle de la «grande dépression». Au Canada, cette interprétation a été remise en question au début des années 1960, notamment par Bertram qui proposa une vision nettement moins sombre du contexte économique des années 1873 à $1896^{6}$. Selon lui, le rythme de croissance du secteur manufacturier canadien, mesuré à l'aide des données de recensements, se rapproche sensiblement de celui enregistré pendant les années 1896 à 1910, établies comme des années de prospérité.

Toutefois, cette remise en question reposait sur l'utilisation de données éclairant des secteurs différents de ceux jusqu'alors examinés. Ainsi, on a remplacé les volumes des activités commerciales et les données démographiques comme les soldes migratoires - qui démontraient l'existence d'un malaise profond entre 1873 et 1896 - par la valeur de la production du secteur manufacturier. Aussi faut-il peutêtre retenir des performances économiques modestes sur certains plans et sur d'autres des progrès indéniables. Les activités forestières québécoises se logent davantage à la première enseigne, c'est-à-dire qu'elles accusent un bilan assez sombre jusqu'en 1890.

\section{La tendance au plafonnement de la production forestière québécoise}

La production de bois équarri connaît durant cette période des moments décisifs. Une grave crise économique frappe le monde occidental en 1873 - au Québec ses effets se font sentir à compter de 1874

\footnotetext{
5 Voir Guy Gaudreau, L'exploitation des forêts publiques au Québec: 1842-1905 (Québec, Institut québécois de recherche sur la culture, 1986).

6 Voir G. W. Bertram, «Economic Growth in Canadian Industry, 1870-1915: the Stapel Model», W. T. Easterbrook, M. H. Watkins, dir., Approaches to Canadian Economic History (Toronto, McClelland and Stewart, 1967), 74-98; G. W. Bertram, «Historical Statistics on Growth and Structure of Manufacturing in Canada, 1870-1957», J. Henripin et A. A. Asimakopoulos, dir., Canadian Political Science Association Conference on Statistics: 1962 and 1963 Papers (Toronto, University of Toronto Press, 1964).
} 
TABLEAU 1

La production de bois équarri, de bois de sciage et de bois à pâte en provenance des terres publiques québécoises, 1870-1905, en millions de pmp ${ }^{1}$

\begin{tabular}{|c|c|c|c|c|c|c|c|c|}
\hline \multirow[t]{2}{*}{ ANNÉE } & \multicolumn{3}{|c|}{ BOIS ÉQUARRI } & \multicolumn{3}{|c|}{ BOIS DE SCIAGE } & \multirow{2}{*}{$\begin{array}{l}\text { BOIS À } \\
\text { PÂTE }\end{array}$} & \multirow{2}{*}{ TOTAL } \\
\hline & Pin & Autres & Total & Pin & Épinette & Total & & \\
\hline $1870-71$ & 60,2 & 1,1 & 61,3 & 293 & 38 & 331 & - & 392 \\
\hline $1871-72$ & 63,2 & 1 & 64,2 & 355 & 43 & 408 & - & 472 \\
\hline $1872-73$ & $49,6^{*}$ & 1,8 & 51,4 & 377 & 86 & 463 & - & 514 \\
\hline $1873-74$ & 45,3 & 10 & 55,3 & 382 & 101 & 483 & - & 538 \\
\hline $1874-75$ & $47,1^{*}$ & 11 & 58,1 & 242 & 58 & 300 & - & 358 \\
\hline $1875-76$ & 65,4 & 8,7 & 74,1 & 201 & 76 & 277 & - & 351 \\
\hline $1876-77$ & 48,1 & 3,1 & 51,2 & 206 & 83 & 289 & - & 340 \\
\hline $1877-78$ & 39 & 2,3 & 41,3 & 170 & 76 & 246 & - & 287 \\
\hline $1878-79$ & 37,9 & 3,1 & 41 & 189 & 60 & 249 & - & 290 \\
\hline $1879-80$ & 19,2 & 1,7 & 20,9 & 287 & 111 & 398 & - & 419 \\
\hline $1880-81$ & 31 & 1,9 & 32,9 & 377 & 116 & 493 & - & 526 \\
\hline $1881-82$ & 48,5 & 1,5 & 50 & 397 & 123 & 520 & - & 570 \\
\hline $1882-83$ & 37,7 & 1 & 38,7 & 391 & 111 & 502 & - & 541 \\
\hline $1883-84$ & 16,1 & 2,2 & 18,3 & 252 & 62 & 314 & - & 332 \\
\hline $1884-85$ & 3 & 1,8 & 4,8 & 324 & 88 & 412 & - & 417 \\
\hline $1885-86$ & 6,4 &, 3 & 6,7 & 344 & 116 & 460 & - & 467 \\
\hline $1886-87$ & 6,3 & 1,9 & 8,2 & 324 & 97 & 421 &, 3 & 430 \\
\hline $1887-88$ & 7,2 & ,7 & 7.9 & 468 & 130 & 598 & 1 & 607 \\
\hline $1888-89$ & 25,8 & 8 & 26,6 & 392 & 103 & 495 & 6 & 528 \\
\hline $1889-90$ & 37,8 & ,9 & 38,7 & $302^{*}$ & 189 & 491 & 4 & 534 \\
\hline $1890-91$ & 9,3 & 2 & 11,3 & 317 & $197 *$ & 514 & 6 & 531 \\
\hline 1891-92 & 15,1 & 6 & 15,7 & 428 & $199 *$ & 627 & 4 & 647 \\
\hline $1892-93$ & 22,9 & ,3 & 23,2 & 370 & 192 & 563 & 4 & 590 \\
\hline $1893-94$ & 1,9 & 1 & 2 & 341 & 213 & 554 & 4 & 560 \\
\hline $1894-95$ & 17,4 &, 5 & 17,9 & 418 & 270 & 688 & 7 & 713 \\
\hline $1895-96$ & 5,6 &, 3 & 5,9 & 381 & 276 & 658 & 2 & 666 \\
\hline $1896-97$ & 15,5 & ,9 & 16,4 & 341 & 360 & 702 & 3 & 721 \\
\hline $1897-98$ & 10,7 & 4,4 & 15,1 & 245 & 323 & 568 & 2 & 585 \\
\hline 1898-99 & 5 & 2,7 & 7,7 & 291 & 309 & 600 & 4 & 612 \\
\hline $1899-00$ &, 8 &, 5 & 1,3 & 340 & 360 & 700 & 2 & 703 \\
\hline $1900-01$ & 7,6 & 1,6 & 9,2 & $307 *$ & 320 & 627 & $156^{* *}$ & 792 \\
\hline $1901-02$ & 6,1 & ,7 & 6,8 & 284 & 288 & 571 & 122 & 700 \\
\hline $1902-03$ & 11,4 & 1,8 & 13,2 & 277 & 377 & 655 & 156 & 824 \\
\hline 1903-04 & 3,1 & 5 & 8,1 & 298 & 431 & 729 & 156 & 893 \\
\hline 1904-05 & & & 3 & 298 & 365 & 663 & 194 & 860 \\
\hline
\end{tabular}

* Donnée corrigée.

** Voir la note 30 .

Source: Rapport du commissaire des Terres de la Couronne de la province de Québec. 
- pour se terminer en 1879. Dans un pareil contexte, on pourrait croire que l'équarrissage, qui n'affichait aucune vitalité particulière durant les années 1860, s'effondre. Hamelin et Roby avec d'autres ont même conclu que cette crise économique internationale marque la fin de la production du bois équarri ${ }^{7}$. Or, cette fin survient plus tard. Le milieu des années 1880 situe plutôt l'amorce du déclin de cette production (voir le tableau 1). De la saison 1873-1874 à celle de 1875-1876, l'équarrissage est même en hausse; il frôle alors le sommet atteint en 1863-1864. Après une seule saison difficile, celle de 1879-1880, cette production rebondit à nouveau pour ensuite s'affaisser littéralement au milieu des années $1880^{8}$. Un soubresaut à la fin de cette décennie pourrait laisser croire à une reprise. Mais il s'agit d'une remontée éphémère; cette production disparaît à toutes fins utiles au début du 20e siècle.

Ces hauts niveaux de production atteints durant la crise internationale étonnent. Sans doute faut-il les associer à la phase intense de construction que connaît alors la Grande-Bretagne ${ }^{9}$, et qui entraîne de fortes exportations de bois équarri entre les années 1875 et 1878 (voir le tableau 2 ). Ces exportations tendent toutefois à baisser au cours des années 1880 .

Par ailleurs, de fortes fluctuations ponctuent la production de bois équarri. D'une année à l'autre, les variations peuvent atteindre 1500 , voire $2000 \%$. Cette évolution syncopée est le fait d'une activité devenue accessoire dans la stratégie des entrepreneurs forestiers, une activité à laquelle ils se prêtent uniquement lorsque le marché les y invite. Le caractère intermittent de cette activité remonte aux années 1860, c'est-à-dire depuis que plusieurs concessionnaires s'adonnent simultanément aux deux types de production: le bois équarri et le bois de sciage $^{10}$.

Contrairement au bois équarri, le bois de sciage a été profondément touché par la crise (voir le tableau 1). La chute des récoltes s'établit à 45\% (272 millions de pieds-mesure de planche en moyenne entre 1874-1875 et $1878-1879$ et 483 millions de pmp en 1873-1874). Le repli des activités de sciage est brutal et la décennie qui suit la crise en est une de consolidation. Même si l'épinette subit une baisse moins accentuée de ses récoltes durant la crise, elle est toujours moins recher-

7 Jean Hamelin et Yves Roby, Histoire économique du Québec, 1851-1896 (Montréal, Fides, 1971), 91; W. T. Easterbrook et H. G. J. Aitken, Canadian Economic History (Toronto, Macmillan, 1970), 204.

8 Lower avait bien identifié le moment où cette activité a périclité en mentionnant la décennie de 1880; voir «The Trade in Square Timber», dans Approaches to..., 47.

9 Voir J. Perry Lewis, Building Cycles and Britain's Growth (Londres, MacMillan, 1965), 316-317. Notons que la tendance nord-américaine ne connaît pas ses sommets en même temps que ceux observés en Grande-Bretagne. Buckley avait déjà signalé ce phénomène, «Urban Building and Real Estate Fluctuations in Canada», CJEPS, 18,1 (1952): 41-62.

${ }_{10}$ A. R. M. Lower, Great-Britain's Woodyard..., 159. 
TABLEAU 2

Évolution des exportations québécoises et ontariennes de bois de sciage et de bois équarri, 1870-1900, en milliers de pmp et en pieds cubes

\begin{tabular}{|c|c|c|c|}
\hline Année & $\begin{array}{l}\text { Planches }^{1} \\
\text { en milliers } \\
\text { de pmp }\end{array}$ & $\begin{array}{l}\text { Madriers }^{2} \\
\text { en milliers } \\
\text { de pmp }\end{array}$ & $\begin{array}{l}\text { Bois équarri }^{3} \\
\text { (en pieds cubes) }\end{array}$ \\
\hline $1870-71$ & 702499 & 184671 & 21809800 \\
\hline $1871-72$ & 751953 & 193353 & 24367240 \\
\hline $1872-73$ & 739034 & 402564 & 21774560 \\
\hline $1873-74$ & 781232 & 487608 & 16853280 \\
\hline $1874-75$ & 454715 & 280167 & 20996960 \\
\hline $1875-76$ & 336110 & 264360 & 17656280 \\
\hline $1876-77$ & 330225 & 290925 & 24694160 \\
\hline $1877-78$ & 353625 & 251631 & 17567640 \\
\hline $1878-79$ & 371546 & 159371 & 7468760 \\
\hline $1879-80$ & 621240 & 215710 & 8777440 \\
\hline $1880-81$ & 579216 & 314440 & 20177920 \\
\hline $1881-82$ & 619001 & 237773 & 11559720 \\
\hline $1882-83$ & 574193 & 296387 & 12926480 \\
\hline $1883-84$ & 614234 & 311710 & 13997200 \\
\hline $1884-85$ & 608299 & 257529 & 9866840 \\
\hline $1885-86$ & 573830 & 349269 & 10466000 \\
\hline $1886-87$ & 556235 & 331273 & 6111240 \\
\hline $1887-88$ & 586242 & 256768 & 7053040 \\
\hline $1888-89$ & 686899 & 245091 & 8650800 \\
\hline $1889-90$ & 674710 & 396459 & 10742640 \\
\hline $1890-91$ & 724670 & 359510 & 8415440 \\
\hline $1891-92$ & 688141 & 295193 & 7146360 \\
\hline $1892-93$ & 816658 & 328246 & 6430640 \\
\hline 1893-94 & 1262352 & 344446 & 6722600 \\
\hline $1894-95$ & 706971 & 319096 & 4690200 \\
\hline $1895-96$ & 756157 & 411351 & 6491440 \\
\hline 1896-97 & 1067069 & 384912 & 6067760 \\
\hline $1897-98$ & 528820 & 495624 & 5814240 \\
\hline 1898-99 & 598290 & 524290 & 6516480 \\
\hline $1899-00^{4}$ & 685367 & 470327 & 5233640 \\
\hline
\end{tabular}

1 Les planches expédiées à l'étranger sont comptabilisées en pmp.

2 Les madriers exportés sont dénombrés en centaines de madriers étalons. Ceux-ci ont 11 pouces de large, 12 pieds de long et deux pouces et demi d'épaisseur; ce qui représente 27,5 pmp par madrier. Chaque centaine de madriers étalons renferme ainsi $2750 \mathrm{pmp}$.

3 Le bois équarri exporté est mesuré en «load». Un load équivaut à 40 pieds cubes.

4 À compter de 1900-1901, les données relatives aux exportations ne sont plus ventilées par province.

Source: Tableaux sur le commerce et la navigation du Canada; Documents de la session. 
chée que le pin. Ce dernier reste encore l'essence par excellence dans les scieries. Toutefois, la performance de la saison 1889-1890, durant laquelle l'abattage de l'épinette double pratiquement par rapport à la saison précédente, laisse présager un changement majeur dans la répartition des essences sciées. Voilà certainement un fait marquant à retenir dans l'évolution de cette production.

L'examen de l'affermage confirme par ailleurs les malaises du secteur forestier qu'annonce le déclin de l'équarrissage combiné au piétinement du sciage. Entre 1874 et 1890 , l'aire québécoise de coupe oscille entre 40000 et 45000 milles carrés. Donc, peu de nouveaux affermages: la piètre qualité des boisés non encore concédés n'y invitait guère.

L'évolution de la demande étrangère traduite par les données relatives aux exportations renforce ce constat relatif au tassement de la production du bois de sciage. L'examen des marchés américain et britannique révèle en effet une stabilisation de la demande jusqu'en $1887^{11}$. Lors de la crise, celle-ci s'est effondrée du côté américain: la quantité de planches acheminées a été réduite de moitié. Aussitôt la crise terminée, les exportations reprennent; elles atteignent alors un volume légèrement inférieur à celui du début des années 1870 pour s'y maintenir par la suite. La demande britannique démontre quant à elle une remarquable stabilité de 1874-1875 à 1888-1889. Par ailleurs, si on observe une chute des arrivées de madriers lors de la crise, c'est en raison de l'insuffisance du point de repère, car les années 1872-1873 et 1873-1874 sont exceptionnelles ${ }^{12}$. Aussi, devons-nous évaluer les exportations de madriers durant la crise en les confrontant à celles enregistrées en 1870 et 1871 . Dans cette perspective, les années 1874 à 1879 laissent deviner une situation beaucoup moins alarmante pour les madriers. En outre, les lendemains de la crise ne s'accompagnent pas, comme c'est le cas pour le marché américain, d'une hausse des expéditions.

Il importe de rappeler que les années 1873 à 1879 en GrandeBretagne se déroulent dans un contexte d'essor de la construction ${ }^{13}$ dont

11 Signalons que le tableau 2 présente l'ensemble des exportations ontariennes et québécoises. Ce choix est nécessaire parce qu'une bonne partie des expéditions québécoises vers les États-Unis transitent par l'Ontario, tandis que la totalité des envois ontariens vers la GrandeBretagne passe par le Québec. Par ailleurs, en insistant sur le niveau des exportations pour justifier un essoufflement de la production québécoise, nous sommes conscient de renouer ainsi avec les auteurs qui décrivaient la période 1873-1896 comme étant celle d'une «grande dépression».

12 Un examen attentif de la valeur des expéditions de madriers au cours de ces deux années démontre que le gonflement des volumes alors exportés ne s'accompagne pas d'une hausse de la valeur de ces expéditions. Autrement dit, la croissance en pmp s'est faite au prix d'une diminution de la valeur moyenne des madriers vendus.

13 Marr et Paterson affirment que ce phénomène est lié à une diminution des investissements britanniques à l'étranger et à un ralentissement de l'émigration britannique qui crée une demande de logements en Grande-Bretagne; Canada: an Economic History (Toronto, Gage, 1980), 281283 . 
profite le secteur forestier québécois. En revanche, la construction s'effondre brutalement aux États-Unis durant cette même période; c'est vers la fin des années 1880 qu'elle y entre à nouveau en effervescence, le sommet se situant entre 1887 et $1889^{14}$. Sans doute faut-il y voir la cause du haut niveau de production de matière ligneuse enregistrée au Québec au cours de la saison 1887-1888.

Durant cette période difficile, même la demande intérieure influence négativement le volume global de la production de bois de sciage. En effet, jusqu'en 1890, on dénombre seulement quelques années animées d'un fort mouvement de construction domiciliaire, soit la fin des années 1880 (voir le tableau 3). Mais pour être appréciable, la hausse de la consommation intérieure de matière ligneuse ne peut compenser la faiblesse de la demande étrangère qui ne se redresse qu'à la fin des années 1880, soit en même temps que les activités de construction au Canada.

TABLEAU 3

Évolution du nombre de permis de construction accordés par la Ville de Montréal, 1870-1904

\begin{tabular}{|c|c|c|c|}
\hline Année & Nombre de permis & Année & Nombre de permis \\
\hline 1870 & 603 & 1888 & 933 \\
1871 & 1060 & 1889 & 1032 \\
1872 & 728 & 1890 & 937 \\
1873 & 724 & 1891 & 778 \\
1874 & 850 & 1892 & 640 \\
1875 & 732 & 1893 & 561 \\
1876 & 463 & 1894 & 382 \\
1877 & 349 & 1895 & 277 \\
1878 & 241 & 1896 & 315 \\
1879 & 240 & 1897 & 408 \\
1880 & 209 & 1898 & 351 \\
1881 & 248 & 1899 & 357 \\
1882 & 231 & 1900 & 331 \\
1883 & 260 & 1901 & 443 \\
1884 & 317 & 1902 & 467 \\
1885 & 429 & 1903 & 581 \\
1886 & 699 & 1904 & 799 \\
1887 & 1032 & & \\
\hline
\end{tabular}

Source: Les rapports de l'inspecteur des bâtisses de la Ville de Montréal.

14 Voir M. Gottlieb, Estimates of Residential Building, United States, 1840-1939 (National Bureau of Economic Research, 1964), 61-62. 


\section{2 - UN NOUVEL ESSOR: 1890-1905}

Les années du tournant du $20^{\mathrm{e}}$ siècle correspondent à la phase de démarrage du bois à pâte. Quoique ce dernier n'affiche pas encore l'importance du bois de sciage, sa poussée est telle qu'on doit déjà le considérer comme le nouveau moteur de l'exploitation forestière. Si le bois de sciage continue de se maintenir comme la principale récolte de matière ligneuse, il connaît par ailleurs des difficultés qui tendent à en stabiliser le volume. Voyons plus en détail les caractéristiques de cette nouvelle période.

\section{Une exploitation plus intensive de la matière ligneuse}

L'évolution de la production de bois de sciage se distingue par des inflexions nombreuses et accentuées (voir le tableau 1). En dépit de ces contractions, se profile une tendance générale à la hausse. Le pin, essence par excellence du bois de sciage, est toutefois en perte de vitesse à compter de 1894-1895.

La croissance de l'ensemble des récoltes, qui demeure modeste, résulte plutôt de la coupe de l'épinette et des autres essences qu'on regroupe avec elle ${ }^{15}$. Entre 1890 et 1905 , les récoltes d'épinettes doublent, passant de 200 millions à 400 millions de pmp.

En 1890, la coupe atteignait 4,75 millions de billots; quinze ans plus tard, elle s'élève à presque 13 millions de billots. En fait, cette multiplication du nombre de billots est la conséquence de la diminution irrégulière mais continue de leur diamètre moyen. Elle correspond par ailleurs à une exploitation encore plus intensive de la matière ligneuse. Le règlement de 1892, par exemple, prévoit la commercialisation de la tête des arbres qu'on laissait pourrir sur place auparavant. On établit à cette fin un droit de coupe pour les billots d'un diamètre inférieur à 12 pouces $^{16}$.

Cette évolution des récoltes de bois de sciage se dégage bien des données afférentes à l'exportation de planches et de madriers (voir le tableau 2). Celle-ci s'intensifie durant les 10 premières années. La comparaison entre les années 1880 et 1890 le démontre à l'évidence: la moyenne annuelle des envois de planches grimpe de 600 millions de pmp à 780, tandis que celle des expéditions de madriers passe de 300 millions de pmp à 400 .

15 Ces essences sont notamment le sapin, le cèdre, la pruche et le cyprès.

16 Les inspecteurs du ministère signalaient dans leurs rapports que les concessionnaires ont profité de l'existence de cette clause pour abattre des pins d'un diamètre à la souche inférieur à 12 pouces, tout en prétendant qu'ils provenaient de la tête d'arbres plus volumineux. 
Le marché britannique reste donc encore bien ouvert. Ne représente-t-il pas à l'échelle québécoise et ontarienne le tiers des exportations de bois de sciage? Compte tenu de la relative proximité des régions forestières québécoises à ce marché, on peut croire que cette proportion est encore plus élevée au Québec qu'en Ontario.

Deux facteurs expliquent l'élargissement de la demande étrangère dans les années 1890: 1) la croissance soutenue de la construction en Grande-Bretagne à partir de 1895 , avec un sommet en 1898 et 1899 ; et 2) les modifications apportées aux tarifs américains frappant l'importation du bois de sciage par les États-Unis et aux tarifs canadiens relatifs à l'exportation de bois brut (c'est-à-dire sur les billots de sciage).

Rappelons brièvement l'historique de ces modifications tarifaires, avant d'en retracer les effets. Après l'abrogation du traité de Réciprocité, le gouvernement du Canada s'empresse de sanctionner un tarif sur l'exportation de billots de sciage bruts afin de décourager les entrepreneurs américains de s'approvisionner au Canada en matière ligneuse pour la transformer aux Etats-Unis. Fixé d'abord à un dollar du 1000 pmp, il subit par la suite quelques ajustements pour être finalement porté à $2 \$$ en $1889^{17}$. Le Canada abolit en 1890 cette mesure tarifaire. Les billots traverseront dès lors la frontière sans entrave tarifaire ni d'un côté ni de l'autre, car les États-Unis accueillent le bois brut en franchise depuis 1870. Cependant, les États-Unis continuent encore d'imposer des droits d'entrée sur le bois de sciage: la taxe américaine sur la planche de pin est abaissée de $2 \$$ à $1 \$$, tandis que celle frappant la planche d'épinette reste inchangée à $2 \$$ du $1000 \mathrm{pmp}^{18}$. Enfin, le bois de sciage de toutes catégories doit finalement être admis en franchise de 1894 à 1897. Des pressions exercées par les exploitants américains ont tôt fait cependant d'amener le rétablissement, en 1897 , d'un tarif fixé à $2 \$$ du $1000 \mathrm{pmp}^{19}$. Le gouvernement ontarien réagit alors à cette mesure en interdisant, à partir de 1898-1900, l'exportation de bois brut provenant des terres publiques. Et le Québec en vient à cette décision en 1910.

Entre 1894 et 1897, les mesures tarifaires rendent donc le bois de sciage canadien beaucoup plus concurrentiel sur le marché américain. Les fortes poussées de 1892-1893 et de 1896-1897 y trouvent leur explication. L'exportation de billots de sciage bruts - phénomène nouveau apparaissant à la fin des années 1880 - contribue à gonfler notablement le volume des exportations de matière ligneuse. La masse de billots bruts exportés représente en effet près de $20 \%$ du volume de planches

17 Voir A. R. M. Lower, North American Assault..., 154-156.

18 Le «McKinley Bill» de 1890 prévoyait de plus une clause (l'article 218) par laquelle des droits supplémentaires seraient prélevés si les pays exportateurs (en l'occurrence le Canada) taxaient son bois brut à l'exportation. Cette clause sera reconduite en 1894 et en 1897.

19 J. E. Defebaugh, History of the Lumber Industry of America (Chicago, The American Lumberman, 1906), 2: 453-457. 
exportées entre 1890 et 1897 . Quant à la chute brutale enregistrée en 1897-1898, elle peut très vraisemblablement être imputée à la réimposition des mesures tarifaires américaines.

À l'aube du $20^{\mathrm{e}}$ siècle, les exportations de bois de sciage perdent de leur importance. En fait, la demande se déplace: les expéditions vers l'étranger fléchissent alors que les récoltes ne cessent d'atteindre de nouveaux sommets. C'est la demande intérieure qui prend alors le relais. En ce début du $20^{\mathrm{e}}$ siècle, l'urbanisation au Canada, et plus particulièrement au Québec et en Ontario, connaît une vigoureuse poussée. L'indice proposé par Buckley pour mesurer les activités de construction canadienne passe de 100 à 332,8 entre 1900 et $1905^{20}$.

\section{Les difficultés du sciage}

Plusieurs auteurs, dont A. R. M. Lower, ont signalé que l'industrie du bois de sciage au tournant du $20^{e}$ siècle entrait dans une phase difficile $^{21}$. Deux symptômes auraient révélé ce malaise de l'industrie du sciage, soit le plafonnement des récoltes et la fermeture des plus grandes scieries. Certaines causes de ce malaise ont été identifiées: carence des approvisionnements (le bois est plus petit et de moins bonne qualité); vive concurrence des producteurs de la côte Ouest et des États américains du Sud; arrivée de nouveaux matériaux de construction. Ces facteurs sont importants, mais ils ne suffisent pas à expliquer la détérioration de la situation du sciage. Une analyse plus poussée encore s'impose pour bien comprendre le malaise qui frappe le sciage à la charnière du $20^{\mathrm{e}}$ siècle.

En premier lieu, il faut admettre que ce malaise a de longs antécédents; en fait, il remonte à la période antérieure. Cette assertion, qui va à l'encontre de ce qui est entendu généralement, provient du fait que notre analyse repose sur des bases différentes de celles utilisées par l'historien qui a le plus marqué l'histoire forestière, Lower. Celui-ci a voulu comprendre l'histoire forestière canadienne dans son ensemble. Une tâche des plus ambitieuses, faut-il reconnaître. Son analyse a reposé néanmoins, pour une bonne part, sur l'exemple ontarien qu'il connaissait mieux. Lower a décrit la période 1870-1900 comme l'âge d'or de l'industrie du bois de sciage dans l'Est du Canada ${ }^{22}$. Cependant, on ne

$20 \mathrm{~K}$. Buckley, loc. cit. Son indice est construit, pour le tournant du $20^{\mathrm{e}}$ siècle, à partir des permis de construction accordés dans quelques villes canadiennes. Il fournit donc une meilleure indication du niveau de la consommation intérieure. Notons néanmoins que les activités de construction sont en effervescence à Montréal à compter de 1900 (voir le tableau 3).

21 A. R. M. Lower, North American Assault..., 200-201; Raoul Blanchard, Le Canada français (Montréal, Fayard, 1960), 129; Paul-André Linteau, René Durocher, Jean-Claude Robert, Histoire du Québec contemporain, 1: De la Confédération à la crise (Montréal, Boréal Express, 1978), 134.

22 A. R. M. Lower, North American Assault..., chapitre 14. 
trouve pas dans ses ouvrages de mesures de l'évolution de la production forestière. À défaut d'un tel outil, il ne pouvait guère constater les difficultés du secteur forestier québécois à partir de la crise qui éclate en 1873.

Les difficultés du sciage ne se manifestent pas d'un seul coup à l'échelle du territoire forestier québécois. Elles touchent d'abord la région du Saint-Maurice, dès $1875^{23}$, et celle du Saguenay-Lac-SaintJean, à compter de 1880. Entre-temps, l'Outaouais, le coeur du Québec forestier du $19^{\mathrm{e}}$ siècle, est pour ainsi dire épargné. Ce n'est qu'à partir de 1890 , on le voit plus loin, que cette région éprouve à son tour des difficultés sérieuses dans le domaine du sciage.

Cela nous amène à croire que ces difficultés du sciage tiennent surtout à un appauvrissement des ressources exploitables que met fort bien en relief le phénomène déjà souligné de la réduction graduelle du diamètre des arbres exploités. L'évolution des volumes physiques de billots de pin et d'épinette montre une diminution de $50 \%$ du contenu moyen des billots d'épinette entre 1888 et 1905 . Cette diminution est d'une amplitude considérable. Elle provient d'une coupe beaucoup plus intensive, c'est-à-dire beaucoup moins sélective. Moins importante est la diminution du diamètre des billes de pin: près de $33 \%$. Ces deux pourcentages sont assurément plus élevés que ceux qu'on obtient entre 1874-1888 24: il faut donc convenir d'une accélération de la baisse du volume moyen des billots à partir de 1890 .

Faire ressortir les effets de cette réduction du volume moyen des billots sur l'industrie du sciage paraît alors essentiel: ils entraînent à coup sûr une majoration des coûts d'exploitation. Bien que l'on assiste, au cours de cette période, à une hausse de l'ensemble des récoltes de bois de sciage, on peut quand même y voir des signes de malaise. En effet, le gonflement de la production ne se fait qu'au prix d'une exploitation intensive et coûteuse de petits billots. Plus concrètement, la diminution des diamètres moyens exerce peut-être ses effets les plus néfastes à l'étape cruciale du tranchage du billot. Bien que nos connaissances sur les techniques utilisées alors dans le sciage souffrent de nombreuses lacunes $^{25}$, nous croyons tout de même pouvoir suggérer la piste de recherche suivante.

Quand le sciage est à son apogée, soit au début des années 1870 , la technique la plus répandue de transformation du billot en planches

\footnotetext{
23 Voir René Hardy, Normand Séguin, Forêt et société..., 75.

24 En Ontario, les volurnes des billes de pin ont diminué d'environ $26 \%$ entre 1874 et 1888 , tandis qu'au Québec, nous avons estimé cette baisse à environ 16\% (voir le tableau 1).

${ }_{25}$ On consultera l'article étoffé de A. J. H. Richardson, «Indications for Research in the History of Wood-Processing Technology», Bulletin of the Association for Preservation of Technology, 10,3 (1974): 35-146.
} 
est celle décrite par Joseph Tassé en $1873^{26}$. Cette technique suppose trois opérations successives. La première est le tranchage du billot dans sa longueur. La deuxième fait intervenir la déligneuse («edger») qui élimine les imperfections et l'écorce demeurées attachées sur les rebords de la planche et lui donne une largeur standard. Une dernière opération consiste à ébouter la planche pour la ramener à une longueur standard. Comme la précédente, cette opération n'a pas connu de modifications majeures depuis un siècle: les deux s'effectuent à l'aide de petites scies circulaires très efficaces et tournant à grande vitesse. Un coup d'oeil sur les machines à l'oeuvre lors du tranchage montre bien toutefois qu'elles s'adaptaient mal à la diminution des volumes. Les scies utilisées pour cette opération ont un mouvement vertical. Elles sont regroupées de manière à trancher simultanément le billot à plusieurs endroits équidistants. Ce premier tranchage dure quelques minutes et doit être repris, car il a laissé intact le centre de la pièce de bois. Le procédé est donc relativement long mais efficace tant que les billes gardent de bonnes dimensions. Par ailleurs, ce procédé implique que la durée totale du tranchage ne dépend pas du volume des billots mais plutôt de leur longueur. On obtient comme résultat une baisse de productivité consécutive à la diminution des volumes, car la quantité de matière ligneuse produite pour une même durée est moindre.

Cette technique de moins en moins productive représente alors un problème pour les entrepreneurs. Le renouvellement de leurs machineries s'impose s'ils veulent ajuster la durée du tranchage en fonction du diamètre des billots. Ont-ils su le faire, et de manière satisfaisante? Nous ne saurions donner une réponse catégorique à cette question. Pourtant, la machine qui aurait pu apporter une solution à leur problème est déjà disponible sur le marché depuis la guerre de Sécession ${ }^{27}$ : la scie à ruban. Lower affirme qu'on la retrouve au Canada au cours des années $1890^{28}$. Son principe de fonctionnement est simple: au lieu de procéder simultanément à plusieurs tranchages, la scie à ruban effectue un seul tranchange à la fois et ce, à une vitesse beaucoup plus rapide. Le temps de cette opération est ainsi proportionnel au volume de la bille. La scie à ruban possède en outre un autre avantage qui favorise sa diffusion: elle permet de réduire substantiellement les pertes de bois imputables à la largeur du trait de $\operatorname{scie}^{29}$. Cependant, des témoignages oraux recueillis auprès de travailleurs contemporains nous invitent à la

26 Joseph Tassé, La Vallée de l'Outaouais (Montréal, 1873), cité par Hamelin et Roby, op. cit., 221-222.

27 Nathan Rosenberg, «America's Rise to Woodworking Leadership», B. Hindle, ed., America's Wooden Age: Aspects of its Early Technology (New York, Sleepy Hallow Restorations, 1975), 47.

28 A. R. M. Lower, North American Assault..., 50.

29 Voir Nathan Rosenberg, loc. cit., 48. Afin d'expliquer l'introduction de la scie à ruban, l'auteur met l'accent sur son avantage en termes de largeur de trait de scie (1/12 de pouce par rapport à $3 / 8$ de pouce pour les autres scies). Ce point est capital et doit être retenu. Toutefois, le gain le plus important demeure, à notre avis, celui de la productivité. 
prudence dans l'appréciation des qualités de la scie à ruban, car il faut tenir compte de sa fragilité. La lame est si fine qu'elle risque à tout moment de se rompre. En somme, le malaise du sciage provient aussi d'un problème technique, lui-même étroitement lié à la réduction graduelle du diamètre moyen des billots de sciage.

\section{L'évolution de la production de bois à pâte}

Le bois à pâte constitue bien le principal moteur de l'exploitation forestière durant cette période. S'il y a augmentation substantielle des récoltes, c'est en raison de l'importance croissante du bois à pâte dans l'ensemble de la production forestière (voir le tableau 1). Il n'est pourtant pas aisé de suivre séparément l'évolution des récoltes de bois à pâte, à cause de leur sous-enregistrement ${ }^{30}$. Par bonheur, il semble bien que les données à partir de la saison 1900-1901 soient utilisables de même que celles de la fin des années 1880 . Estimée en fonction de ces points de repère, l'augmentation de la production paraît vertigineuse: 4 millions de pmp en 1890 et 156 millions 11 ans plus tard (voir le tableau 1). Cette croissance rapide montre bien l'importance du bois à pâte en fin de période. Il représente déjà, entre 1901-1902 et 1904-1905, 24\% des récoltes de bois de sciage. Autrement dit, le bois à pâte compte à l'aube du $20^{\mathrm{e}}$ siècle pour environ $18 \%$ de l'ensemble de la valeur de la production forestière, car, en plus du bois de sciage, il faut tenir compte des bois divers et des pièces de bois équarri encore déclarées (le tout représente $6 \%$ du total des droits de coupe).

C'est sur les marchés étrangers que le bois à pâte trouve ses principaux débouchés. Tout comme les concessionnaires déclarant du bois équarri, ceux qui s'activent dans la production du bois à pâte fournissent moins de la moitié du total des expéditions vers l'étranger. La plus grande part vient des producteurs exploitant des terres privées. Il ne faudrait pas déduire cependant que les débouchés du bois à pâte cueilli sur les terres publiques sont essentiellement étrangers. Une ventilation des informations dans les rapports annuels du commissaire des Terres de la Couronne au début du siècle nous permet d'affirmer que $68 \%$ des récoltes sont transformées au Canada.

Une véritable mutation du secteur forestier se précise à travers ces données et sera complétée durant les années 1920 avec la prédominance du bois à pâte. Des amendements à la réglementation relative au dia-

30 À la fin du $19^{\mathrm{e}}$ siècle, une partie substantielle des récoltes de bois à pâte est enregistrée sous la rubrique billot de sciage d'épinette sans qu'on puisse distinguer les deux types de récolte. Ce sous-enregistrement est tel qu'il est en partie responsable de la hausse rapide des billots de sciage d'épinette durant cette période. Le phénomène a été examiné plus en détail dans Guy Gaudreau, L'exploitation des forêts... (Québec, Institut québécois de recherche sur la culture, 1986), 45-47. 
mètre minimal des arbres exploitables rendent possible un changement non seulement quantitatif mais aussi qualitatif. Des essences jusqu'alors ignorées, et même des arbres jugés trop petits pour le bois de sciage, sont maintenant recherchés. Il n'est pas surprenant alors de voir la superficie totale affermée s'étendre considérablement à compter de $1898^{31}$ : plus de 25000 milles carrés de nouvelles concessions s'ajoutent aux anciens territoires de coupe entre 1898 et 1905 . L'industrie des pâtes et papiers a rendu ces nouveaux lieux d'abattage alléchants en augmentant leur valeur commerciale. Aussi peut-on observer une forte hausse des prix des nouvelles concessions. Mieux encore, le bois à pâte a rehaussé la valeur des vieilles concessions qui ont longtemps alimenté le sciage.

\section{3 - LA DIMENSION RÉGIONALE DES ACTIVITÉS FORESTIERES}

Les grandes tendances de l'évolution des récoltes s'appliquentelles à l'histoire de chaque région forestière ${ }^{32}$ ? Assurément non. Il convient d'insister encore sur ce phénomène en évoquant des faits tirés des deux phases.

Le sciage enregistre entre 1874 et 1890 , une forte progression en Outaouais supérieur avec une hausse de $40 \%{ }^{33}$. Compte tenu des conditions du marché, cette performance est appréciable. Toutefois, on ne peut en dire autant de trois autres régions pour lesquelles on note une baisse des récoltes. Dans la région du Saint-Maurice, on peut parler d'une plongée brutale des récoltes de bois de sciage. La chute de production est de l'ordre de $60 \%$ et provoque la fermeture de chantiers, des faillites ou des abandons d'affaires. La réduction de la superficie concédée témoigne d'ailleurs de ce recul (voir le tableau 4). Au Saguenay-Lac-Saint-Jean et dans l'Outaouais inférieur, la baisse de la production, évaluée en volume physique et non en billots, est moins brutale et peut être estimée à 30 et $15 \%$ respectivement. Quant à la région de la Rive-Sud, on peut considérer sa performance comme moyenne, dans la mesure où elle enregistre une hausse de $10 \%$ de ses récoltes.

31 Dans un premier temps l'industrie papetière s'est accaparée de vieilles concessions avec lesquelles on avait produit beaucoup de bois de sciage mais qui étaient devenues moins rentables.

32 Nous avons retenu cinq régions forestières pour les besoins de l'analyse. Ce choix est rendu nécessaire par l'obligation de conserver des territoires dont les frontières sont immuables et naturelles. Pour plus de détails, voir Guy Gaudreau, L'exploitation des forêts... (Québec, Institut québécois de recherche sur la culture, 1986), 78-85.

33 La prudence est de rigueur dans ces analyses des activités forestières régionales d'avant 1890 , car il faut tenir compte de la diminution graduelle du format des arbres exploités et de l'impossibilité de convertir systématiquement les billots en pmp à l'échelle régionale (les risques de distorsion par des variantes régionales mal connues seraient trop considérables). Toutefois, on peut considérer que les billots déclarés en 1890 ont déjà diminué de 20\% par rapport à 1873-1874. Pour retrouver les données utilisées dans cet article pour l'analyse des récoltes régionales, on consultera l'appendice 3 de notre thèse de doctorat. 
TABLEAU 4

Évolution des récoltes forestières dans les 5 régions du Québec, 1874-1890

\begin{tabular}{|l|c|c|c|c|}
\hline & $1874-75$ & $1879-80$ & $1883-84$ & $1886-87$ \\
& $1878-79$ & $1882-83$ & $1885-86$ & $1889-90$ \\
\hline Outaouais supérieur & & & & \\
superficie en \% & 40,8 & 40,8 & 43,5 & 44,8 \\
droits de coupe en \%1 & 60,0 & 68,2 & 68,5 & 71,4 \\
intensité de la coupe & 1,47 & 1,67 & 1,57 & 1,59 \\
Outaouais inférieur & & & & \\
superficie en \% & 12,1 & 12,8 & 11,0 & 12,2 \\
droits de coupe en \% & 12,0 & 10,3 & 11,2 & 11,0 \\
intensité de la coupe & 0,99 & 0,80 & 1,02 & 0,90 \\
Saint-Maurice & & & & \\
superficie en \% & 23,2 & 21,5 & 20,0 & 17,0 \\
droits de coupe en \% & 11,4 & 7,6 & 6,3 & 4,4 \\
intensité de la coupe & 0,49 & 0,35 & 0,32 & 0,26 \\
Saguenay-Lac-Saint-Jean & & & & \\
superficie en \% & 10,5 & 10,2 & 10,4 & 11,9 \\
droits de coupe en \% & 7,2 & 4,6 & 4,6 & 4,4 \\
intensité de la coupe & 0,69 & 0,45 & 0,44 & 0,37 \\
Rive-Sud & & & & \\
superficie en \% & 13,3 & 14,6 & 15,1 & 14,1 \\
droits de coupe en \% & 9,4 & 9,2 & 9,4 & 8,8 \\
intensité de la coupe & 0,71 & 0,63 & 0,62 & 0,62 \\
\hline
\end{tabular}

1 En pourcentage sur le total québécois.

2 L'intensité de la coupe est calculée en divisant le pourcentage des droits de coupe régionaux par le pourcentage de la superficie régionale.

Source: Rapport du commissaire des Terres de la Couronne de la province de Québec.

Le portrait est tout autre après 1890 . La situation s'est détériorée en Outaouais supérieur, puisqu'on ne constate aucune hausse de production (voir le tableau 5). Sans l'annexion du bassin de la Lièvre en 1900 - lequel était rattaché auparavant à la région de l'Outaouais inférieur - une chute des volumes récoltés y aurait sans doute été observée. En revanche, deux régions, celles du Saint-Maurice et de la Rive-Sud, connaissent des activités plus soutenues. Si on totalise le bois de sciage et le bois à pâte, la croissance de la production forestière est de $200 \%$ pour la région de la Rive-Sud et de $425 \%$ pour la région du SaintMaurice. Sans obtenir une performance équivalente, l'Outaouais inférieur affiche un bilan nettement positif. La coupe du bois de sciage et du bois à pâte y croît au rythme de l'évolution de la production à l'échelle québécoise; la région génère à peu près le même pourcentage de droits de coupe en 1890 et en 1905 (voir le tableau 5). Ce volume d'activité 
TABLEAU 5

Évolution des récoltes forestières dans les 5 régions du Québec, 1890-1905

\begin{tabular}{|c|c|c|c|c|c|}
\hline & \begin{tabular}{|l|}
$1890-91$ \\
$1892-93$
\end{tabular} & \begin{tabular}{|l|}
$1893-94$ \\
$1894-95$
\end{tabular} & \begin{tabular}{|l|}
$1895-96$ \\
$1897-98$
\end{tabular} & \begin{tabular}{|l|}
$1898-99$ \\
$1900-01$
\end{tabular} & \begin{tabular}{|l}
$1901-02$ \\
$1904-05$
\end{tabular} \\
\hline $\begin{array}{l}\text { Outaouais supérieur } \\
\text { superficie en } \%^{1} \\
\text { droits de coupe en } \%^{1} \\
\text { intensité de la coupe }\end{array}$ & $\begin{array}{c}44,3 \\
64,1 \\
1,45\end{array}$ & \begin{tabular}{|c|}
43,2 \\
60,8 \\
1,41
\end{tabular} & \begin{tabular}{|c}
40,1 \\
52,5 \\
1,31
\end{tabular} & \begin{tabular}{|c}
38,5 \\
53,0 \\
1,38
\end{tabular} & \begin{tabular}{|c|}
39,2 \\
45,7 \\
1,17
\end{tabular} \\
\hline $\begin{array}{l}\text { Outaouais inférieur } \\
\text { superficie en } \% \\
\text { droits de coupe en } \% \\
\text { intensité de la coupe }\end{array}$ & $\begin{array}{c}12,4 \\
12,8 \\
1,03\end{array}$ & $\begin{array}{c}10,9 \\
11,2 \\
1,03\end{array}$ & $\begin{array}{c}11,6 \\
12,8 \\
1,10\end{array}$ & \begin{tabular}{|c}
8,8 \\
13,4 \\
1,52
\end{tabular} & \begin{tabular}{|c|}
5,2 \\
11,7 \\
2,25
\end{tabular} \\
\hline $\begin{array}{l}\text { Saint-Maurice } \\
\text { superficie en \% } \\
\text { droits de coupe en \% } \\
\text { intensité de la coupe }\end{array}$ & \begin{tabular}{|c|}
18,7 \\
6,31 \\
0,34
\end{tabular} & $\begin{array}{c}18,1 \\
10,7 \\
0,59\end{array}$ & $\begin{array}{c}18,0 \\
13,1 \\
0,73\end{array}$ & \begin{tabular}{|c}
20,2 \\
12,1 \\
0,60
\end{tabular} & \begin{tabular}{|c}
21,8 \\
18,9 \\
0,87
\end{tabular} \\
\hline $\begin{array}{l}\text { Saguenay-Lac-Saint-Jean } \\
\text { superficie en } \% \\
\text { droits de coupe en } \% \\
\text { intensité de la coupe }\end{array}$ & \begin{tabular}{|c|}
10,8 \\
4,9 \\
0,45
\end{tabular} & $\begin{array}{c}11,9 \\
4,9 \\
0,41\end{array}$ & $\begin{array}{c}14,7 \\
4,6 \\
0,31\end{array}$ & \begin{tabular}{|c}
16,3 \\
3,5 \\
0,21
\end{tabular} & \begin{tabular}{|c|}
17,1 \\
5,8 \\
0,34
\end{tabular} \\
\hline $\begin{array}{l}\text { Rive-Sud } \\
\text { superficie en \% } \\
\text { droits de coupe en \% } \\
\text { intensité de la coupe }\end{array}$ & $\begin{array}{c}13,9 \\
11,3 \\
0,81\end{array}$ & \begin{tabular}{|c}
15,8 \\
12,4 \\
0,78
\end{tabular} & $\begin{array}{c}14,3 \\
17,0 \\
1,19\end{array}$ & $\begin{array}{c}16,1 \\
18,0 \\
1,12\end{array}$ & $\begin{array}{c}16,7 \\
17,9 \\
1,07\end{array}$ \\
\hline
\end{tabular}

1 En pourcentage sur le total québécois.

2 L'intensité de la coupe est calculée en divisant le pourcentage des droits de coupe régionaux par le pourcentage de la superficie régionale.

Source: Rapport du commissaire des Terres de la Couronne de la province de Québec.

est appréciable, si l'on retient qu'en 1900 la région perd le bassin de la Lièvre. Quant au Saguenay-Lac-St-Jean, les récoltes font des gains modestes $(40 \%)$, en dépit d'une forte augmentation des superficies affermées.

\section{Les débouchés commerciaux des régions forestières}

Comment expliquer de si fortes différences régionales? Les débouchés commerciaux en sont sûrement une des principales causes. Faute de données précises sur ce sujet ${ }^{34}$, l'évolution des marchés amé-

\footnotetext{
34 L'historiographie est en effet très pauvre sur cette question. Lower avait déjà identifié grossièrement les débouchés commerciaux de certaines régions, mais celles-ci ne correspondent pas à notre découpage spatial. Voir North American Assault..., 166-168.
} 
ricain et britannique sera comparée avec le développement des activités forestières de chacune des régions. Appliqué entre 1856 et 1890, ce procédé comparatif grossier permet d'entrevoir vers quel marché principal sont dirigées les récoltes de chaque région.

Le Saguenay-Lac-Saint-Jean représente sans doute le cas le plus simple: son développement se confond avec le rythme de la demande britannique de madriers ${ }^{35}$. Pendant les 17 premières années, la coupe y progresse lentement puis, soudain, la production s'accélère avec la hausse rapide des ventes de madriers. Durant la crise, la situation est beaucoup moins catastrophique que partout ailleurs au Québec. Les récoltes d'épinette, bien qu'elles régressent momentanément, demeurent vives. A telle enseigne que, durant la saison 1877-1878, on dépasse, pour cette essence, le niveau record enregistré en 1872-1873 ${ }^{36}$. Aussi, les droits de coupe tirés du sciage sont légèrement en hausse durant la crise. Toutefois, à la fin des années 1880 , une cassure se produit sans que la demande britannique ne soit en cause. On peut ici avancer l'hypothèse que l'épuisement des grosses épinettes, utilisées pour la production de madrier, en soit responsable.

Jusqu'à la crise la Rive-Sud semble connaître la même situation que le Saguenay-Lac-Saint-Jean. En effet, son développement est d'abord lent, puis les progrès deviennent spectaculaires en 1873 et en 1874. Sauf pour sa partie ouest, sa position géographique facilite l'établissement de liens commerciaux avec la Grande-Bretagne. La crise toutefois y sévit avec plus d'intensité qu'au Saguenay-Lac-Saint-Jean; néanmoins, la Rive-Sud s'en tire mieux que les trois autres régions. La reprise au lendemain de la crise y est beaucoup plus forte que dans ces dernières. Au Saguenay-Lac-Saint-Jean, la reprise est nulle, ce qui tend à démontrer l'existence de rapports commerciaux étroits avec le marché britannique. Pour la même période, la Rive-Sud enregistre une croissance de 50\%, ce qui indique une articulation au marché américain.

La région du Saint-Maurice est liée davantage à l'évolution de la demande américaine. La hausse de la coupe y est plus régulière jusqu'à la crise: c'est dire qu'on n'y observe aucune croissance vigoureuse des récoltes. La crise, par contre, s'abat brusquement sur la région et, dans les années 1880, tout se passe comme si les entrepreneurs, privés peu à peu de pins, étaient incapables de tirer profit de son immense réserve d'épinettes. S'agit-il encore d'un épuisement des ressources exploitables? Probablement. La région ressemble de toute manière à une terre

\footnotetext{
35 Cela est d'ailleurs confirmé par Arthur Buies, Le Saguenay et le bassin du Lac SaintJean (Québec, 1896), 148-149. Troisième édition.

36 À l'inverse des autres régions, c'est en 1872-1873, plutôt qu'en 1873-1874, que le Saguenay-Lac-Saint-Jean enregistre sa plus forte production d'épinettes avant la crise.
} 
inhospitalière ${ }^{37}$ : une des plus importantes familles d'entrepreneurs de la région, les Baptist, va étendre ses activités ailleurs en formant une société qui sera active dans l'Outaouais ${ }^{38}$.

De toutes les régions, l'Outaouais inférieur est celle dont le rythme de développement est le plus équivoque. Les statistiques comparatives sont insuffisantes et empêchent de préciser son principal débouché.

Il y a plus à dire heureusement sur l'Outaouais supérieur. L'immensité de son territoire de coupe (voir le tableau 4), et les dimensions cyclopéennes de ses récoltes reposent vraisemblablement sur de multiples articulations au marché. Le bois équarri dont elle produit l'essentiel des récoltes est expédié en Grande-Bretagne. Mais l'évolution de ce marché ne paraît pas marquer de manière déterminante son développement. Il en est autrement du marché américain. La consommation américaine de matière ligneuse explique à la fois la poussée de ses récoltes jusqu'en 1874 et les effets désastreux de la crise. La reprise est en outre à la mesure du marché américain: les gains déclarés sont alors de $100 \%$. Néanmoins, la croissance vigoureuse de sa production jusqu'en 1874 demeure sans doute caractéristique d'une réponse à la demande intérieure ${ }^{39}$. C'est pourquoi il faut également convenir du rôle du marché intérieur dans le développement de cette région.

L'année 1890 marque une rupture très nette dans le développement des activités forestières régionales. Jusqu'à cette date, se maintient la tendance à la concentration géographique des récoltes forestières. En dépit d'une relative stabilité de la distribution régionale des superficies affermées, le regroupement des droits de coupe par région indique une répartition de plus en plus inégale de la valeur de la production. En 1890 , la part de l'Outaouais supérieur atteint $71 \%$, un sommet pour toute la deuxième moitié du $19^{\mathrm{e}}$ siècle. On imagine peut-être mal les conséquences de ce haut niveau de production dans une même région. Les autres régions ressemblent alors à des zones forestières marginales.

Mais, par la suite, le processus s'inverse (voir les tableaux 4 et 5). Deux autres régions, celle du Saint-Maurice et celle de la Rive-Sud, prennent une part accrue dans les récoltes de matière ligneuse de sorte que, au début du $20^{\mathrm{e}}$ siècle, l'Outaouais supérieur ne génère plus que $45 \%$ des droits de coupe.

Ce nouvel élan est tributaire de l'émergence du bois à pâte. Avec son arrivée, le lent processus d'épuisement des ressources prend une

\footnotetext{
37 Buies avait observé la baisse abrupte de l'exploitation forestière dans le Saint-Maurice dans les années 1880. Voir L'Outaouais supérieur (Québec, 1889), 198-199.

38 Cette société porte le nom de «Ottawa Lumber Co.», et ses «lettres patentes» sont accordées en 1886. Voir la Gazette officielle de Québec, 1886, 2017.

39 Quoique cela reste à être démontré, on peut suggérer aussi l'apport de l'Outaouais inférieur au marché intérieur, en raison notamment de sa proximité à la région montréalaise.
} 
autre tournure. Sous le règne du bois de sciage, le pin est l'essence par excellence. Mais il s'épuise et est remplacé dans plusieurs régions par les récoltes d'épinette. Ce remplacement d'ailleurs ne se fait pas sans heurts. À preuve, le blocage de la région du St-Maurice, à partir de la crise, et celui de la région du Saguenay-Lac-Saint-Jean durant les années 1880. L'épinette devient l'essence la plus recherchée, ressuscitant ainsi des régions dépourvues ou dégarnies de pins.

Entre 1890 et 1905, la région de la Rive-Sud triple pratiquement ses récoltes d'épinette, et celle du Saint-Maurice en fait autant. A l'aube du $20^{\mathrm{e}}$ siècle, cette région produit plus de $50 \%$ des récoltes de bois à pâte. Ces récoltes occupent $48 \%$ du volume des activités forestières de la région du Saint-Maurice. Un pourcentage légèrement inférieur est observé dans la région du Saguenay-Lac-Saint-Jean (40\%).

\section{CONCLUSION}

La période 1874-1890 est certes une période de transition dans l'exploitation des forêts publiques au Québec. D'abord parce que cette période marque un temps d'arrêt dans la croissance des récoltes québécoises: le manque de vigueur de la demande intérieure, adossé à la stabilisation de la demande étrangère, ne permet pas une majoration des productions à l'échelle québécoise, et la crise a contribué à cette situation. Ensuite, parce que des signes avant-coureurs de mutation apparaissent déjà: si le pin domine encore les essences exploitées, la performance de l'épinette, en fin de période, laisse présager déjà l'essor du bois à pâte. Certaines régions, comme celle du Saint-Maurice, ont épuisé leurs pins et grosses épinettes et se retrouvent en difficulté. Cela permet à la région de l'Outaouais supérieur d'occuper une plus grande place dans le secteur forestier.

Globalement, le secteur forestier québécois connaît une phase fort différente de sa contrepartie ontarienne. La croissance des récoltes y est rapide, en raison notamment d'une expansion de l'aire de coupe. Cela nous amène à suggérer que le débat sur la grande dépression devrait davantage tenir compte de la différence des performances d'un même secteur entre les provinces.

L'émergence du bois à pâte provoque une rupture dans le secteur forestier, à compter de 1890. Rupture en terme d'évolution des récoltes: celles-ci sont résolument à la hausse. Rupture aussi sur le plan de la localisation des activités: la région de l'Outaouais supérieur cède peu à peu sa place dominante à des régions riches en épinettes.

Un certain malaise, manifesté par une tendance au plafonnement des volumes, est perceptible dans l'industrie du sciage. On réussit toujours à produire des quantités appréciables de pmp, mais au prix de quantités sans cesse grandissantes de billots. La technique du tranchage 
du billot est mal adaptée à ce contexte et toutes les manipulations additionnelles des billes tendent à faire de l'industrie du sciage une industrie où les profits sont moins alléchants. C'est dans ce contexte que se situe l'arrivée, au $20^{\mathrm{e}}$ siècle, des francophones dans l'industrie québécoise du sciage: il ne s'agit plus d'un secteur très rentable. D'autres recherches permettront d'approfondir cette histoire.

Dernier élément à retenir: la variété des performances régionales. On évoquera à titre d'exemple la bonne performance de la région du Saguenay-Lac-Saint-Jean durant la crise. Celle-ci est loin d'avoir affecté les régions également. Cela étant admis, le secteur forestier québécois apparaît comme un ensemble disparate que façonnent plusieurs types d'articulation au marché et - autre facteur capital - un éventail divers de potentiel ligneux s'épuisant à des rythmes différenciés.

\section{ANNEXE 1}

Le tableau-synthèse de l'évolution des récoltes de matière ligneuse entre 1874 et 1905 s'appuie sur une série de traitements des données brutes. La plupart d'entre eux ont déjà été exposés en détail dans un ouvrage antérieur ${ }^{40}$. Aussi, est-il inutile d'y revenir. Par contre, il importe d'expliciter certaines opérations mises au point afin de compléter ce travail méthodologique.

La plus importante demeure sans conteste l'évaluation en volume physique des récoltes de bois de sciage entre 1874 et 1887 , période au cours de laquelle les données officielles comptabilisent le bois de sciage en billot et non en unité de mesure faisant référence à un volume physique fixe ${ }^{41}$. Nous avions déjà proposé une estimation pour ces années, mais nous souhaitons reconsidérer ces éléments et revoir toutes les récoltes de sciage d'avant 1887.

Il convient d'abord d'exposer succinctement la méthode que nous avions adoptée. Essentiellement, il s'agissait de se servir de l'évolution annuelle du contenu moyen des billes de pin ontariennes - lequel est connu pour la première fois à partir de 1874 - et de l'adapter aux données québécoises. Affichant 151,6 pmp en 1873-1874, ce contenu moyen s'établit à 109,4 pmp en 1887-1888 (voir le tableau A). Ces chiffres ne doivent pas étonner. Pour une des régions ontariennes, soit la zone outaouaise, on a relevé 170,7 pmp en $1873-1874$ et 118,3 en 1887-1888. En fait, la réduction graduelle des volumes moyens est une

\footnotetext{
40 Voir Guy Gaudreau, L'exploitation des forêts publiques au Québec: 1842-1905 (Québec, Institut québécois de recherche sur la culture, 1986), 40-66.

${ }_{41}$ Le billot est une simple unité comptable, car son diamètre est variable et inconnu d'une saison à l'autre. Cela étant, il est impossible d'évaluer sur cette base les volumes récoltés.
} 
des caractéristiques fondamentales des activités forestières. Ce phénomène résulte d'un rythme d'exploitation de la forêt plus rapide que son rythme de croissance. Il marque également les activités forestières québécoises. C'est pourquoi nous avions appliqué aux premières données québécoises fournissant le contenu moyen des billes, soit celles de 18871888 , le rythme de croissance ontarien (car nous fonctionnons à rebours, c'est-à-dire de 1887-1888 vers les années antérieures). Nous disons le rythme de croissance et non le contenu moyen lui-même ${ }^{42}$.

Il faut admettre que ce procédé était trop mécanique. Appliquer intégralement le rythme ontarien provoque sans doute des distorsions. D'une saison à l'autre, le contenu moyen ontarien peut décroître tandis qu'il est temporairement en hausse au Québec. Faut-il alors remettre en question le taux de croissance observé, soit $38 \%$ sur une période de 15 ans?

Dans le but d'apporter des éléments nouveaux, nous avons examiné plus en profondeur les données québécoises et ontariennes. Ces premières, par exemple, présentent un aspect, pourtant digne d'intérêt, qui nous avait jusqu'alors échappé. Deux catégories de billes de pin sont consignées entre 1868-1869 et 1886-1887: celle d'un diamètre égal ou supérieur à 17 pouces, et celle d'un diamètre inférieur. Or, la proportion du nombre de billots déclarés entre les deux catégories constitue un point de repère grossier en vue de définir un rythme de croissance spécifiquement québécois. En effet, si la proportion des plus grosses billes diminue par rapport aux petites, le contenu moyen de l'ensemble risque fort de chuter. Voyons alors comment, vues sous cet angle nouveau, les récoltes de pin ont évoluées (voir le tableau B). Les chiffres révèlent clairement une réduction très sensible du diamètre. Avec un ratio de 0,85 en 1873-1874, il est sûr que le contenu moyen des billots est supérieur à celui de 1886-1887 alors que ceux de petit diamètre sont presque deux fois plus nombreux que les grosses billes $(0,52)$.

Autre observation: le rythme québécois de réduction ou de croissance est différent. Durant la crise, les diamètres rapetissent en Ontario tandis qu'au Québec les données semblent démontrer une majoration des volumes par rapport à 1873-1874. En effet, on abat alors plus de gros billots que de petits. Cela n'est peut-être pas si étonnant: la crise sévissant beaucoup plus durement au Québec (-45\%) qu'en Ontario $(-25 \%)$, les entrepreneurs ont sans doute tendance à se procurer les billes les plus rentables.

42 La table de conversion ontarienne est différente de celle adoptée au Québec en 1888. C'est pourquoi, le contenu moyen des billes de pin blanc est, en 1887-1888, de 109,9 pmp en Ontario et de $137,8 \mathrm{pmp}$ au Québec. 
Derrière ces chiffres un phénomène essentiel se dessine: plus il y a croissance du nombre de billots, plus le rythme de réduction des diamètres des billes risque de s'accélérer. Or, encore ici, la situation québécoise diffère. Dans la province voisine, on observe un taux de croissance de plus de $120 \%$ entre 1874 et 1887. Mais au Québec, celuici oscille autour de $15 \%$.

Il faut donc écarter l'idée d'appliquer mécaniquement le taux de croissance ontarien. D'abord, les données de crise constituent fort probablement une période au cours de laquelle on enregistre une hausse du contenu moyen. Ensuite, le taux de 38\% demeure excessif si l'on tient compte de la faible hausse du nombre de billots récoltés entre 1874 et 1887. Nous suggérons en remplacement: un taux d'environ $15 \%$ s'appliquant entre les années 1880 et 1887 selon la progression des ratios calculés au tableau B, et une hausse momentanée des volumes durant la crise. Les résultats sont alignés au tableau C. En outre, comme il n'y a aucune raison de croire que les billots d'épinette seraient à l'abri de cette réduction des diamètres, nous avons appliqué les mêmes pourcentages et les mêmes rythmes à ces récoltes.

Comptabilisées en pmp, les récoltes de sciage ne posent aucun problème d'équivalence. Il en est autrement néanmoins du bois équarri et du bois à pâte. Pour ce dernier, l'équivalence en usage dans les règlements d'alors a été conservé, soit $600 \mathrm{pmp}=$ une corde de bois à pâte ${ }^{43}$. Quant au bois équarri, son unité de mesure usuelle, le pied cube, a été ramenée en pmp, sur la base de $12 \mathrm{pmp}=1$ pied cube.

TABLEAU A

Évolution du contenu moyen des billes de pins blancs abattues sur les terres publiques ontariennes 1874-1887, en pmp

\begin{tabular}{|l|l|l|}
\hline $1873-74: 151,6$ & $1878-79: 142,5$ & $1883-84: 118,5$ \\
$1874-75: 161,6$ & $1879-80: 130,9$ & $1884-85: 118,5$ \\
$1875-76: 143,2$ & $1880-81: 124,4$ & $1885-86: 122,7$ \\
$1876-77: 139,7$ & $1881-82: 116,0$ & $1886-87: 122,1$ \\
$1877-78: 138,2$ & $1882-83: 111,9$ & $1887-88: 109,9$ \\
\hline
\end{tabular}

Source: Rapport annuel du commissaire des Terres de la Couronne de l'Ontario.

43 Voir le règlement adopté le 1er juin 1901, paru dans la Gazette officielle du Québec, $1901,1342$. 
TABLEAU B

Évolution des récoltes de billes de pin en provenance des terres publiques québécoises, 1874-1887, en billots

\begin{tabular}{|l|l|l|l|}
\hline $\begin{array}{l}\text { Saison } \\
\text { d'exploitation }\end{array}$ & Billes $>17$ po & Billes $<17$ po & $\frac{(\text { Billes }>17 \text { po })}{\div \text { (Billes }<17 \text { po })}$ \\
\hline $1873-74$ & 1099285 & 1288583 &, 85 \\
$1874-75$ & 789016 & 677331 & 1,17 \\
$1875-76$ & 682640 & 501336 & 1,36 \\
$1876-77$ & 711001 & 465641 & 1,53 \\
$1877-78$ & 570251 & 462629 & 1,23 \\
$1878-79$ & 617354 & 561691 & 1,10 \\
$1879-80$ & 848954 & 942919 &, 90 \\
$1880-81$ & 021168 & 1397790 &, 73 \\
$1881-82$ & 977535 & 1634451 &, 60 \\
$1882-83$ & 943971 & 1698677 &, 56 \\
$1883-84$ & 688697 & 1015177 &, 66 \\
$1884-85$ & 861339 & 1325759 &, 65 \\
$1885-86$ & 888994 & 1497620 &, 59 \\
$1886-87$ & 787766 & 1507246 &, 52 \\
\hline
\end{tabular}

Source: Rapport annuel du commissaire des Terres de la Couronne du Québec.

TABLEAU C

Estimation du contenu moyen des billes de pin et d'épinette abattues sur les terres publiques québécoises, 1874-1888, en pmp

\begin{tabular}{|l|l|l|}
\hline Saison d'exploitation & Pin & Épinette \\
\hline $1887-88$ & 137,8 & 79,3 \\
$1886-87$ & 141 & 81 \\
$1885-86$ & 144 & 83 \\
$1884-85$ & 148 & 85 \\
$1883-84$ & 148 & 85 \\
$1882-83$ & 148 & 85 \\
$1881-82$ & 152 & 87 \\
$1880-81$ & 156 & 89 \\
$1879-80$ & 160 & 92 \\
$1878-79$ & 160 & 92 \\
$1877-78$ & 165 & 95 \\
$1876-77$ & 175 & 100 \\
$1875-76$ & 170 & 97 \\
$1874-75$ & 165 & 95 \\
$1873-74$ & 160 & 92 \\
\hline
\end{tabular}

